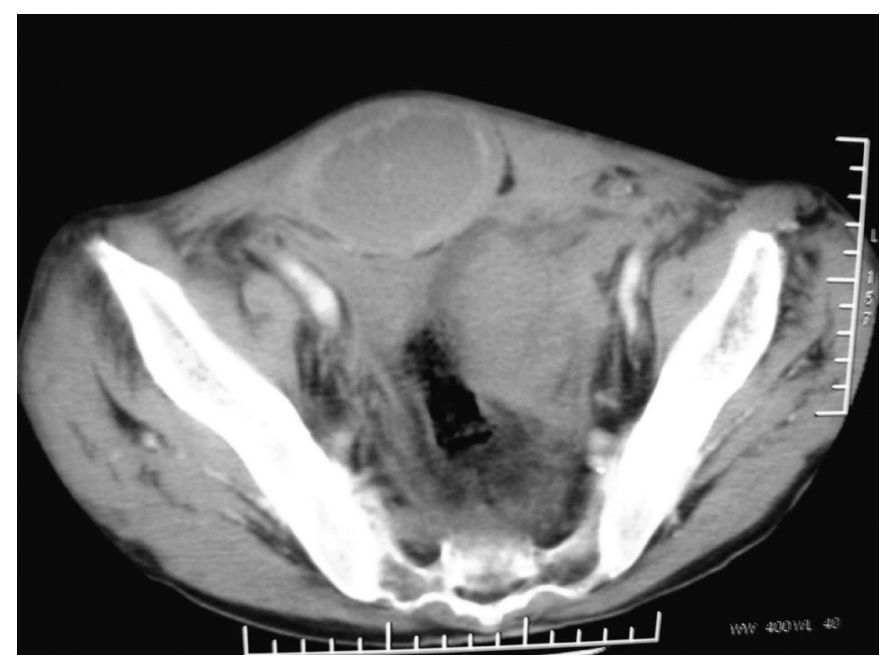

Fig. 1. Tomografía computerizada abdominal con masa a nivel de recto anterior derecho con líquido libre en pelvis.

co. En 1996 se creó una clasificación según las características de la imagen de la TC $(5,6)$, siendo nuestro caso un tipo III con sangrado en peritoneo y espacio prevesical presentando éstos mayor gravedad. La punción-aspiración ha desaparecido como maniobra diagnóstica siendo como tratamiento también ineficaz aumentando el riesgo de infección. Se recomienda generalmente actitud conservadora con analgesia, reversión de la anticoagulación, colocación de bolsas de hielo y reposición de la volemia, valorando cirugía si existe repercusión hemodinámica, compresión de estructuras vitales o infección del hematoma (7-10).

En conclusión, debe tenerse en cuenta esta patología en pacientes con tratamiento anticoagulante y dolor abdominal, realizando estudios de imagen precozmente para valorar la extensión e iniciar tratamiento oportuno.

I. M. Arias Miranda, R. García Fanjul ${ }^{1}$, C. López de Mesa ${ }^{1}$, M. E. González García ${ }^{2}$

Servicios de Medicina Interna, ${ }^{1}$ Medicina Intensiva $y{ }^{2}$ Hematología-Hemoterapia. Hospital de Cabueñes. Gijón

1. Acea Nebril B, Taboada Filgueira L, Sanchez Gonzalez F, Freire Rodriguez D, Fraguela Marina J, Aguirrezabalaga Gonzalez J, Gomez Freijoso C. Acute abdomen in anticoagulated patients. Its assessment and the surgical indications. Rev Clin Esp 1995; 195: 463-7.

2. Pelayo Salas A, Garces Guallart MC, Sola Marti R, Vinas Salas J, Perez Ruiz L. A spontaneous hematoma of the rectus abdominis sheath. Rev Clin Esp 1999; 199: 647-9.

3. Maharaj D, Ramdass M, Teelucksingh S, Perry A, Naraynsingh V. Rectus sheath haematoma: a new set of diagnostic features. Postgrad Med J 2002; 78: 755-6.

4. Barry TL, Butt J, Awad ZT. Spontaneous rectus sheath hematoma and an anterior pelvic hematoma as a complication of anticoagulation. Am J Gastroenterol 2000; 95: 3327-8.

5. Berna JD, García-Medina V, Guirao J, García-Medina J. Rectus sheath hematoma: diagnostic classification by CT. Abdom Imaging 1996; 21 : $62-4$.

6. Fukuda T, Sakamoto I, Kohzaki S, Uetani M, Mori M, Fujimoto T, et al. Spontaneous rectus sheath hematomas: clinical and radiological features. Abdom Imaging 1996; 21: 58-61.

7. Berna JD, Zuazu I, Madrigal M, García-Medina V, Fernández C, Guirado F. Conservative treatment of large rectus sheath hematoma in patients undergoing anticoagulant therapy. Abdom Imaging 2000; 25 : 230-4.
8. O'Mara MS, Semins H, Hathaway D, Caushaj PF. Abdominal compartment syndrome as a consequence of rectus sheath hematoma. Am Surg 2003; 69: 975-7.

9. García Fanjul RM, Antuña Braña MT, Lapuerta Irigoyen JA, Gutiérrez Fernández MJ. Hematoma abdominal secundario al empleo de nadroparina. An Med Interna (Madrid) 2004; 21: 612.

10. Gómez de la Torre R, Milla Crespo A, Cadenas F, Fernández Bustamante J, Vázquez Castañón M. Hematoma retroperitoneal espontáneo inducido por enoxiparina a dosis terapéuticas. An Med Interna (Madrid) 2003; $20: 386-7$

\section{Sarcoidosis y déficit selectivo de IgA}

\section{Sr. Director:}

La sarcoidosis es una enfermedad granulomatosa sistémica de causa desconocida que afecta especialmente a individuos jóvenes. Dentro de las alteraciones de laboratorio presentes en la sarcoidosis destaca la hipergammaglobulinemia policlonal. La coexistencia de un déficit aislado de IgA y sarcoidosis ha sido comunicado previamente de manera ocasional y en casos puntuales (1-3). Presentamos el caso de una mujer joven diagnosticada simultáneamente de sarcoidosis aguda y déficit selectivo de $\operatorname{IgA}$, que recientemente hemos tenido la oportunidad de estudiar.

Se trata de una mujer de 21 años sin antecedentes personales de interés que consultó por artralgias y lesiones de eritema nodoso en piernas. Entre los datos de laboratorio el hemograma y bioquímica completa fueron normales, siendo la velocidad de sedimentación globular de $39 \mathrm{~mm}$ a la primera hora con proteína C reactiva normal. Una radiografía de torax fue normal. La prueba de Mantoux y la serología a toxoplasma, citomegalovirus y virus de Epstein-Baar fueron negativas. Los niveles de enzima convertidora de angiotensina (ECA) se encontraron repetidamente elevados: $81,7,73,4$ y $65 \mathrm{U} / \mathrm{L}$. La gammagrafía con galio detectó depósito del trazador a nivel uveal y parotideo sin captación a nivel hiliar pulmonar, sugerente de sarcoidosis en estadio inicial con afectación glandular pero no hiliar. Un TAC toracoabdominal demostró un resto tímico en espacio prevascular anterior con parenquima pulmonar normal sin adenopatías mediastínicas ni axilares y ligera esplenomegalia sin adenopatías retroperitoneales. Las pruebas funcionales respiratorias fueron normales. Los niveles de TSH fueron normales. Un proteinograma fue normal y la determinación de inmunoglobulinas detectó un déficit confirmado de IgA 5,6 mg/dl (70-400) con niveles normales de IgG $1.536 \mathrm{mg} / \mathrm{dl}$ e IgM $252 \mathrm{mg} / \mathrm{dl}$. El resultado de poblaciones linfocitarias fue normal y la determinación de factor reumatoide, niveles de complemento C3 y C4 y autoanticuerpos (ANA, antiDNA; anti-Sm, anti-RNP, anti-Ro, anti-La y SCl-70) fueron igualmente normales o negativos. Se realizó tratamiento sintomático (AINE) en las primeras 4 semanas y a los 18 meses de seguimiento la paciente permanece asintomática y no ha presentado ningún proceso infeccioso o de otra naturaleza asociado.

El déficit selectivo de IgA es la inmunodeficiencia primaria más frecuente con una incidencia aproximada de 1 de cada 600 individuos de origen europeo. Existe una gran diferencia en la frecuencia de presentación entre la población blanca-caucasiana y asiática lo que viene a apoyar una predisposición genética. Se ha reportado una frecuencia desde 1:170 en España (4), inusualmente alta, hasta 1:18500 en Japón. El criterio diagnóstico definitivo de un déficit de $\operatorname{IgA}$ es una persona mayor de 4 años que tiene $\operatorname{IgA}$ sérica menor de $7 \mathrm{mg} / \mathrm{dL}$ con $\mathrm{IgG}$ e IgM séricas normales. 
Aunque la gran mayoría de las personas con déficit aislado de IgA están asintomáticas existe una mayor incidencia de infecciones respiratorias de repetición, diarrea crónica y enfermedades autoinmunes. A nivel gastrointestinal se asocia especialmente con infecciones por Giardia lamblia y con la enfermedad celíaca. También existe una asociación significativa entre el déficit aislado de IgA y enfermedades autoinmunes especialmente el lupus eritematoso sistémico y artritis reumatoide. La incidencia de asma y de otras enfermedades atópicas es igualmente más alta en los pacientes con déficit de IgA. También se han comunicado casos asociados a tiroiditis de Hashimoto, enfermedad de Still, anemia perniciosa, miastenia gravis, vitiligo, dermatomiositis, anemia hemolitica, síndrome de Sjögren, purpura trombopenica idiópatica y diabetes tipo 1 . La coexistencia del déficit de $\operatorname{IgA}$ con sarcoidosis también ha sido notificada previamente de manera ocasional y en casos aislados (1-3).

En la sarcoidosis hay una hiperactivación de la inmunidad humoral con una hipergammaglobulinemia de tipo policlonal, que se puede ver en los periodos de actividad pero también en los casos inactivos. El papel de esta aumento policlonal de inmunoglobulinas en la patogénesis de la sarcoidosis no esta claro: puede ser secundario a la presencia de linfocitos $\mathrm{T}$ activados durante la formación del granuloma o bien que tuviera algun papel en la formación del granuloma formando parte de los inmunocomplejos circulantes. Otros estudios han señalado que en la sarcoidosis activa los niveles séricos de IgG e IgA son significativamente más bajos, aunque dentro de los límites normales, que en los casos inactivos, lo cual puede ser debido a su acumulación en las lesiones sarcoideas activas o a que formen parte de los inmunocomplejos circulantes durante las fases de actividad de la enfermedad (5).

Algunos casos de déficit aislado de IgA desarrollan inmunodeficiencia variable común (IDVC) con el tiempo. Por otra parte también se han publicado casos de sarcoidosis asociados con inmunodeficiencia variable común $(6,7)$. La posible presencia de este alteración inmunológica debería ser sospechada en pacientes con sarcoidosis que presentan infecciones recurrentes y no tienen hipergammaglobulinemia. El mecanismo de la aparente relación entre las dos entidades es desconocido, aunque se han propuesto dos posibilidades; que la IDVC predispusiera a infecciones que fueran el agente responsable del desarrollo de la sarcoidosis o que ambas entidades esten asociados con alteraciones similares en las celulas $\mathrm{T}$.

El tratamiento de la deficiencia de IgA se centra en la profilaxis y tratamiento de las infecciones. La administración de inmunoglobulinas o plasma es peligroso por el peligro de desarrollar anticuerpos frente a IgA. En los pacientes con déficit de IgA que precisen transfusiones lo ideal es que sólo recibieran sangre de donantes deficientes de $\operatorname{IgA}$.

\section{López Gómez, P. Gómez Avivar'1, M. A. López Ruz, J. Jiménez Alonso}

Servicio de Medicina Interna. ' Sección de Dermatología. Hospital Universitario Virgen de las Nieves. Granada

1. Rubinstein I, Baum GL, Hiss Y. Selective IgA deficiency and sarcoidosis. Chest 1985; 88: 160.

2. Thomas LL, Alberts C, Pegels JG, Balk AG, von dem Borne AE. Sarcoidosis associated with autoimmune thrombocytopenia and selective $\operatorname{IgA}$ deficiency. Scand J Haematol 1982; 28: 357-359.

3. Rubinstein I, Baum GL. Selective IgA deficiency associated with recurrent sinopulmonary infections in sarcoidosis. Eur J Respir Dis 1984; 65: 550-552.

4. Pereira LF, Sapiña AM, Arroyo J, Viñuelas J, Bardají RM, Prieto L. Prevalence of selective IgA deficiency in Spain: more than we thought. Blood 1997; 90: 893
5. Cagatay T, Bilir M, Gulbaran M, Papila C, Cagatay P. The immunoglobulin and complement levels in the active pulmonary sarcoidosis. Kobe $\mathrm{J}$ Med Sci 2003; 49: 99-106.

6. Sutor G, Fabel H. Sarcoidosis and common variable immunodeficiency. A case of a malignant course of sarcoidosis in conjunction with severe impairment of the cellular and humoral immune system. Respiration 2000; 67: 204-208.

7. Fasano MB, Sullivan KE, Sarpong SB, Wood RA, Jones SM, Johns CJ et al. Sarcoidosis and common variable immunodeficiency. Report of 8 cases and review of the literature. Medicine 1996; 75: 251-261.

\section{Exacerbación aguda de un caso de neumonitis intersticial descamativa tras biopsia pulmonar por videotoracoscopia}

\section{Sr. Director:}

La neumonitis intersticial descamativa (NID) es una enfermedad inflamatoria pulmonar de presentación rara, de etiología desconoci$\mathrm{da}$, que se ha relacionado con agentes virales y con el humo del tabaco (1), aunque su patogenia es también desconocida, se han implicado mecanismos immunopatológicos. Se caracteriza histológicamente por acumulo de macrófagos en los alvéolos pulmonares, afecta mas al varón, sobretodo en la cuarta y quinta década de la vida, radiológicamente se muestra con opacificación en vidrio deslustrado, bilateral, simétrico y sobretodo en las zonas inferiores del pulmón, presentando buena respuesta a los esteroides $(2,3)$.

Nosotros describimos un caso de NID que se exacerbó tras la práctica de biopsia pulmonar por videotoracoscopia.

Pacienta varón, de 70 años de edad, exfumador hasta hace dos meses de 102 paquetes de cigarrillos, con historia de tos y disnea de esfuerzos desde hace muchos años, síntoma este ultimo que progresivamente ha venido en aumento. En agosto del pasado año ingresa en nuestro hospital por presentar aumento de la tos, disnea de reposo, astenia, y febrícula, se le practicó estudio radiológico y tomografía axial computarizada de tórax visualizándose imagen de infiltrados en vidrios deslustrados de forma parcheada, diseminados por ambos campos pulmonares, engrosamiento de septos interlobulares, y del intersticio intralobulillar, la gasometría arterial era de Po2 $42 \mathrm{~mm}, \mathrm{Pco} 234 \mathrm{~mm}, \mathrm{Ph} 7,34$, el estudio funcional respiratorio mostraba CV $1750 \mathrm{cc}$, (49\%), FEV 1200 cc $(50 \%), \mathrm{FEV} / \mathrm{CV} 71 \%$. Se inició tratamiento con prednisona a dosis de $0,5 \mathrm{mg}$ por kilo de peso, mejorando el paciente progresivamente y presentando disminución ostensible de la sintomatología, desapareciendo la disnea de reposo y solo presentándola cuando caminaba rápido, el estudio radiológico también mejoró, disminuyendo el infiltrado intersticial, la gasometría arterial basal era de Po2 60 mm, Pco2 42 mm, Ph 7,36. Posteriormente se le practicó videotoracoscopia izquierda con obtención de tres muestras de biopsia pulmonar del lobar superior y del inferior cuyo resultado histológico muestra abundantes macrófagos alveolares, pas positivo con leve engrosamiento fibroso en los septos alveolares y ligero infiltrado linfocitario, algunas zonas quísticas rodeadas de fibrosis, siendo diagnosticado de NID. Al segundo día de la intervención el paciente comenzó con empeoramiento de su estado general, disnea de reposo, ortopnea, taquipnea, en la radiografía de tórax practicada se observaba mayor infiltración intersticial, y la gasometría arterial respirando aire ambiental era de Po2 $44 \mathrm{~mm}$ Pco2 $34 \mathrm{~mm}$, se descartó que el fallo respiratorio fuese debido a complicaciones de la intervención quirúrgica en 